Rev. Biol. Trop., 47(3): 535-538, 1999

www.ucr.ac.cr www.ots.ac.cr www.ots.duke.edu

\title{
Parasitismo entre especies (Diptera, Hymenoptera) en los nidos de Stictia signata (Hymenoptera: Sphecidae)
}

\author{
Julio A. Genaro \\ Museo Nacional de Historia Natural, Obispo \#61, esquina a Oficios, Plaza de Armas, Habana Vieja 10100, Cuba. Fax \\ 53762 0353. E-mail: cocuyo@mnhnc.inf.cu
}

Recibido 24-IX-1998. Corregido 26-II-1999. Aceptado 12-III-1999.

\begin{abstract}
Stictia signata is one of the most frequently observed sand wasps in the Cuban keys and coasts. Females build their nests in the sand and supply them with flies to feed offspring. Here, I describe the behavior of two species, Liohippelates n. sp. near collusor (Diptera: Chloropidae) and Hexacola sp. (Hymenoptera: Eucoilidae), which enter the nests of S. signata. The observations were carried out from 1989 through 1991 in Caimito beach, Southern Havana province, Cuba. Liohippelates inhabited $100 \%$ of the nests. Its necrofagous larvae fed on the remnants of prey left by the larva of $S$. signata, without affecting the larva. Only in one case did the larva show signs of mortality because, apart from the high number of immature cleptoparasites, there were 53 adult flies feeding on prey body fluids. Hexacola sp. parasitized the larvae of Lionippelates within the cells. In spite of high cleptoparasitism, the sphecid population remained high during the observation years.
\end{abstract}

Key words: Stictia signata, sphecid wasps, prey, Hexacola sp, parasitoid, Liohippelates n.sp. near collusor, cleptoparasite.

Stictia signata (L.) es uno de los esfécidos de mayor tamaño, mejor distribuido y más abundante en Cuba. Durante la época reproductiva las hembras construyen los nidos en el suelo, donde depositan el huevo y las presas (dípteros) que alimentan a la larva. La entrada del nido permanece cerrada en períodos de inactividad para evitar la entrada de parásitos (Evans 1966, Post 1981, Martin y Martin 1990, Sánchez et al. 1990). Existen dípteros clorópidos que penetran a los nidos de avispas solitarias porque sus larvas necrófagas se alimentan de los restos de las presas, dejadas por la larva de la avispa (Evans 1966, Evans et al. 1974). Por otra parte, existen himenópteros eucoúlidos del género Hexacola, parasitoides de los clorópidos del género Liohippelates (como Hippelates en publicaciones anteriores) (Mulla 1962, Legner y Bay 1965, Legner et al. 1966, Eskafi y Legner 1974). Sin embargo, no conozco in- teracciones interespecíficas, de este tipo, en el interior del nido de un esfécido.

A continuación se describe la conducta cleptoparásita de Liohippelates n. sp. circa collusor (Townsend) (Diptera: Chloropidae) en los nidos de $S$. signata y el comportamiento de Hexacola sp. (Hymenoptera: Eucoilidae) como parasitoide de los clorópidos en el interior de los nidos. Se presentan las presas con que $S$. signata aprovisionó el nido y se discuten las posibles causas que originan y mantienen la asociación entre las poblaciones de las tres especies.

\section{MATERIALES Y MÉTODOS}

El estudio se realizó en playa Caimito, sur de la provincia de La Habana, Cuba, mediante viajes intermitentes durante todo el año, desde 
1989 hasta 1991. S. signata nidificó a lo largo de $1 \mathrm{~km}$ de costa, con un área de 4-5 m de ancho, con arena gruesa, donde predominaron las plantas Canavalia maritima (Aubl.) Thouars (Fabaceae) y la gramínea Paspalum sp.

Los nidos fueron excavados para recoger en pomos plásticos el contenido de la celdilla, observarla en el laboratorio y obtener la emergencia de los parásitos. Los capullos de Liohippelates $\mathrm{n}$. sp circa collusor fueron medidos con un micrómetro ocular. Los ejemplares estudiados están depositados en la colección del Museo Nacional de Historia Natural, La Habana. Los clorópidos y eucoílidos están además depositados en el United States National Museum y eucoílidos en la colección de G. Nordlander (Swedish University of Agricultural Sciences).

\section{RESULTADOS}

Características de los nidos y las presas de $S$. signata: Esta avispa construyó los nidos en arena gruesa, con la entrada fundamentalmente en áreas desnudas o con vegetación baja y aislada. Los nidos estuvieron constituídos por un túnel, generalmente oblícuo, o con giros, hasta terminar en una celdilla. Presentaron siempre un cierre externo. A diferencia de otras poblaciones conespecíficas (Evans 1966, Post 1981, Martin y Martin 1990) no realizó cierre interno. La longitud de 62 nidos varió desde 14 hasta $52 \mathrm{~cm}(\mathrm{x}=25.2 \mathrm{~cm}, \mathrm{DE}=6.8)$ y las celdillas estuvieron a una profundidad promedio de $14.5 \mathrm{~cm}$ (DE=4.0, gama: 8.0-29.3 cm).

Los nidos fueron aprovisionados con las siguientes especies de moscas. Entre paréntesis aparece el número y el sexo de las presas, cuando pudo determinarse. Stratiomyidae: Hermetia illucens (L.) (27@, 2907), Hedriodiscus dorsalis (Fabr.) (2@, $\left.50^{7}\right)$, Odontomyia rufipes Loew $\left(31 \bigcirc, 260^{\top}\right)$. Tabanidae: Tabanus lineola Fabr. (17@, 20 ). Syrphidae: Ornidia obesa (Fabr.) $\left(40 \bigcirc, 410^{7}\right)$, Palpada vinetorum (Fabr.) (34@, 33 $\left.\bigcirc^{7}\right)$, P. albifrons (Wiedemann) (7@, $\left.60^{7}\right)$, Meromacrus ruficrus (Wiedemann) $\left(10^{\prime}\right)$, Copestylum pallens (Wiedemann) (2Q, $10^{7}$ ). Muscidae: Stomoxys calcitrans (L.) (2),
Graphomyia sp. (4). Calliphoridae: Cochliomyia macellaria (Fabr.) $\left(18 \bigcirc, 110^{7}\right)$, Synthesiomyia nudiseta (Wulp) (1), Lucinia (Phaenicia) sp. prob. eximia (Wiedemann) $\left(3 \bigcirc, 10^{7}\right)$, Phaenicia spp. (13). Tachinidae: Archytas sp (5). Sarcophagidae: Peckia praeceps (Wiedemann) (11). La longitud promedio de las presas fue de $11.0 \mathrm{~mm}(\mathrm{DE}=2.2$; gama:5.0-15.0 mm; $\mathrm{N}=373$ ). S. signata estuvo activa en la etapa reproductiva durante todo el año.

Conducta de Liohippelates $\mathrm{n}$. sp circa collusor: Los clorópidos del género Liohippelates son conocidos en Cuba con el nombre de guasasas y tienen importancia médica, al provocar enfermedades oftalmológicas, por sus habitos de visitar las secreciones acuíferas de los ojos.

Las moscas siempre volaron en el área de nidos de S. signata. Al arribar una avispa al nido, se desplazaron hacia la entrada. Mientras $S$. signata excavó el cierre externo fue observada desde plantas pequeñas, piedras o el sustrato por las hembras de Liohippelates que generalmente tuvieron el abdómen engrosado, por la presencia de huevos. Hasta 10 individuos permanecieron alrededor de la avispa, penetrando junto a ella a través del túnel, para llegar a la celdilla y ovipositar. En ocasiones hubo interacciones en la abertura del nido, pero debido al alto número de moscas y a su tamaño pequeño, el esfécido optó por acelerar la entrada.

Dentro de la celdilla, los clorópidos ovipositaron sobre las presas. Al nacer, las larvas se alimentaron de los restos de las presas de la larva de Stictia. Por esto, siempre estuvieron hacia el fondo de la celdilla, donde se acumulan los desechos. Esta conducta necrófaga, que no afecta el desarrollo de la larva ha sido mencionada además por Evans (1966) y Evans et al. (1974).

El $100 \%$ de los nidos abiertos tuvo larvas de Liohippelates. Sobre los restos de las presas consumidas, acumuladas en el fondo de la celdilla conté en ocasiones, más de 100 larvas de diferentes estadios. Sólo en un nido que tuvo además 53 Liohippelates adultos, observé una larva poco saludable, destinada a morir. El resto de los nidos tuvieron la larva próxima a 
pupar, coexistiendo con las larvas de las moscas cleptoparásitas. Liohippelates adultos se alimentaron de los fluidos corporales de las presas frescas, lo que posiblemente les permitió permanecer más tiempo en el interior del nido.

Conducta de Hexacola sp.: Como Liohippelates, las hembras de Hexacola sp. estuvieron cerca de las entradas de los nidos de $S$. signata, esperando la apertura del cierre para pasar al interior. Dentro de las celdillas parasitaron las larvas del clorópido. La longitud promedio de 67 capullos de Hexacola sp. fue de $2.5 \mathrm{~mm}$ ( $\mathrm{DE}=0.2$, gama: 2.0-3.2 $\mathrm{mm}$ ).

Para salir del nido, Liohippelates y Hexacola subieron a través del túnel y se mantuvieron cerca de la entrada, esperando la llegada de la avispa que destapó el cierre. La hembras de Liohippelates salieron en grupos y rápidamente. Los movimientos de Hexacola siempre fueron más lentos y su número menor.

En una ocasión observé sobre la arena, el apareamiento de esta especie. El macho montó a la hembra y sobre ella movió las antenas hacia arriba y abajo, sin tocar las del otro sexo. Es probable que las cópulas siempre ocurran en esta área, a donde acuden los machos para encontrar a las hembras vírgenes que nacen en las celdillas y emergen desde el interior de los nidos de $S$. signata.

En el área de nidificación de S. signata, junto a los nidos activos, observé entradas cortas, debido a la excavación reiterada de una hembra en varios lugares muy próximos, sin adentrarse en el sustrato. Estas cavidades fueron comunes y aunque su origen y función no está clara, ambos parásitos permanecieron mucho tiempo penetrando a estos agujeros, por lo que entretuvieron a los parásitos.

A partir del contenido de la celdillas obtuve en el laboratorio 116 capullos de Liohioppelates, de los cuales emergerieron 46 Hexacola, mostrando un $39.6 \%$ de parasitismo.

\section{DISCUSIÓN}

De acuerdo a las interacciones ecológicas que se producen en las especies (Halliday,
1993), entre S. signata y Liohippelates ocurrió parasitismo y dentro de este cleptoparasitismo, ya que las larvas de la mosca, e incluso los adultos obtuvieron su alimento del destinado a la larva de la avispa. Hexacola se comportó como un parasitoide porque se desarrolló en el interior de las larvas de Liohippelates, matándolas. En la relación Stictia-Hexacola hubo mutualismo ya que ambos se beneficiaron: $\mathrm{He}$ xacola encontró en el interior de los nidos de Stictia a su hospedero y Stictia logró menos moscas cleptoparásitas en su celdilla.

$\mathrm{El}$ aprovisonamiento progresivo de los nidos de S. signata, o sea el suplemento continuo de presas a la larva, desde su nacimiento hasta el desarrollo completo, mantuvo las relaciones interespecíficas en esta población, a través de varias generaciones al año. El número de indivíduos que conformó la población de Stictia fue elevado durante años, a pesar de estar cleptoparasitados el $100 \%$ de los nidos, lo que demuestra que la conducta desplegada por estas especies dentro de los nidos, no afectó a la descendencia (con excepción de un caso, donde el número de cleptoparásitos fue muy elevado). Posiblemente para los esfécidos se elevó el costo energético en la captura de las presas, teniendo que cazar más moscas que las que depredarían otras Stictia de una población donde no ocurra el cleptoparasitismo.

Estudios sobre la estructura del nido en especies del género Stictia, y S. signata en otras regiones, señalan la presencia de un cierre interno, además del externo (Evans 1966, Evans y Mattews 1974, Mattews et al. 1981, Sheehan 1984). La ausencia de cierre interno, en la población estudiada, posibilitó el desplazamiento de los parásitos dentro del nido, los cuales desarrollaron mecanísmos, favorecidos por su tamaño pequeño para entrar y salir, dependiendo sólo de la apertura del cierre externo.

\section{AGRADECIMIENTOS}

Agradezco a los especialistas relacionados a continuación, por nombrar las especies estudiadas: G. Garcés (BIOECO, Santiago de Cuba), 
N. Woodley y R. Gagne (Systematic Entomology Laboratory, USDA) presas de Stictia; C.W. Sabrosky (Systematic Entomology Laboratory, USDA) Liohippelates; G. Nordlander (Swedish University of Agricultural Sciences) Hexacola; P. Herrera (Instituto de Ecología y Sistemática) plantas. G. Nordlander y A. S. Menke amablemente enviaron literatura. Doy gracias a J.L. Fontenla por la lectura crítica del manuscrito.

\section{RESUMEN}

S. signata es una de las avispas de la arena más frecuentemente observada en los cayos y las costas de Cuba. Las hembras construyen los nidos en la arena y los abastecen con moscas, para alimentar a la descendencia. Se describe la conducta de dos especies: Liohippelates $\mathrm{n}$. sp. circa collusor (Diptera: Chloropidae) y Hexacola sp. (Hymenoptera: Eucoilidae) para penetrar al interior de los nidos de $S$. signata. Las observaciones se efectuaron durante 1989 hasta 1991, en playa Caimito, sur de la provincia de La Habana, Cuba. Liohippelates cleptoparasitó el 100\% de los nidos. Sus larvas necrófagas se alimentaron de los restos de las presas dejadas por la larva de $S$. signata, sin afectarla. Sólo en un caso la larva mostró signos de mortalidad, porque además del número alto de cleptoparásitos inmaduros, habían 53 moscas adultas alimentándose de los fluidos corporales de las presas. Hexacola sp. fue un parasitoide de las larvas de Liohippelates, en el interior de las celdillas. A pesar del elevado cleptoparasitismo, la población del esfécido se mantuvo elevada durante los años de observación.

\section{REFERENCIAS}

Assem, J. van den 1969. Reproductive behavior of Pseudeucoila bochei (Hymenoptera: Cynipidae). I. Description of courtship behavior. Netherlands J. Zool. 19: 641-648.

Eskafi, F.M. \& E.F. Legner. 1974. Descriptions of immature stages of the cynipid Hexacola sp. near webste$r i$ (Eucoilinae: Hymenoptera), a larval-pupal parasite of Hippelates eye gnats (Diptera: Chloropidae). Canadian Entomol. 106: 1043-1048.
Evans, H.E. 1966. The comparative ethology and evolution of the sand wasps. Harvard University, Cambridge, Massachusetts. $526 \mathrm{p}$.

Evans, H.E. \& R.W. Matthews. 1974. Observations on the nesting behavior of South American sand wasps (Hymenoptera). Biotropica 6: 130-134.

Evans, H. E.; R.W. Matthews \& E. McCallan. 1974. Observations on the nesting behavior of Rubrica surinamensis (De Geer) (Hymenoptera, Sphecidae). Psyche 81: 334-352.

Halliday, T. 1993. Evolutionary ecology. Pp. 307-369. En: P. Skelton (ed.) Evolution. A biological and paleontological approach. Addison-Wesley Publishing Company.

Legner, E.F. \& E.C. Bay. 1965. Predatory and parasitic agents attacking the Hippelates pusio complex in Puerto Rico. J. Agric. Univ. Puerto Rico 49: 377-385.

Legner, E.F.; E.C. Bay \& T.H. Farr. 1966. Parasitic and predacious agents affecting the Hippelates pusio complex in Jamaica and Trinidad. Canadian Entomol. 98: 28-33.

Martin, W.F. \& R.F. Martin. 1990. Reproduction of the sand wasps Stictia signata (L.) and Bicyrtes variegata (Olivier) (Hymenoptera: Sphecidae) on the Caribbean coast of Quintana Roo, México. Pan-Pacific Entomol. 66:71-78.

Mathews, R.W.; R.A. Saunders \& J.R. Mathews. 1981. Nesting behavior of the sand wasp Stictia maculata (Hymenoptera: Sphecidae) in Costa Rica. J. Kansas Entomol. Soc. 54: 249-254.

Mulla, M.S. 1962. Recovery of a cynipoid parasite from Hippelates pupae. Mosquito News 22: 301-302.

Post, D. 1981. Observations on female nesting and male behavior of Stictia signata (Hymenoptera: Sphecidae) in Brazil. Rev. Biol. Trop. 29: 105-113.

Sánchez, C.; R. Alayo \& V. Berovides. 1990. Conducta de nidificación de Stictia signata (L.) (Hymenoptera: Sphecidae). Poeyana 400: 1-10.

Sheehan, W. 1984. Nesting biology of the sand wasp Stictia heros (Hymenoptera: Sphecidae: Nyssoninae) in Costa Rica. J. Kansas Entomol. Soc. 57: 377-386. 